\title{
Local flow at plate edge during water entry
}

Cite as: Phys. Fluids 32, 072103 (2020); https://doi.org/10.1063/5.0013914

Submitted: 16 May 2020 . Accepted: 29 June 2020 . Published Online: 13 July 2020

Shi-Yan Sun (孙士艳), and G. X. Wu (吴国雄) (D)
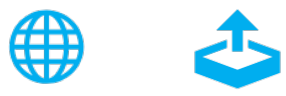

Export Citation

\section{ARTICLES YOU MAY BE INTERESTED IN}

On respiratory droplets and face masks

Physics of Fluids 32, 063303 (2020); https://doi.org/10.1063/5.0015044

Propulsive performance and flow-field characteristics of a jellyfish-like ornithopter with asymmetric pitching motion

Physics of Fluids 32, 071904 (2020); https://doi.org/10.1063/5.0010938

The translational and rotational motions of a cylindrical particle in a granular shear flow inside a split bottom Couette cell

Physics of Fluids 32, 073310 (2020); https://doi.org/10.1063/5.0015175

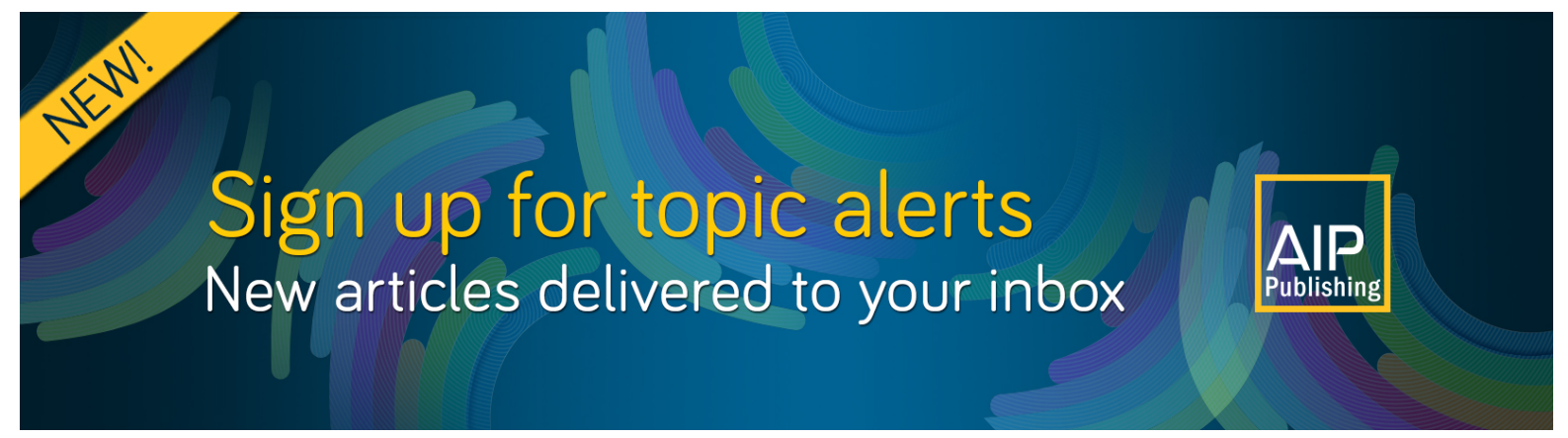




\title{
Local flow at plate edge during water entry
}

\author{
Cite as: Phys. Fluids 32, 072103 (2020); doi: 10.1063/5.0013914 \\ Submitted: 16 May 2020 - Accepted: 29 June 2020 • \\ Published Online: 13 July 2020
}

Shi-Yan Sun (孙士艳) $)^{7}$ and G. X. Wu (吴国雄) ${ }^{2, a)}$ (D)

\begin{abstract}
AFFILIATIONS
${ }^{1}$ School of Naval Architecture and Ocean Engineering, Jiangsu University of Science and Technology, Zhenjiang 212003, China

${ }^{2}$ Department of Mechanical Engineering, University College London, Torrington Place, London WCIE 7JE, United Kingdom
\end{abstract}

a) Author to whom correspondence should be addressed: g.wu@ucl.ac.uk

\begin{abstract}
The local flow near the edge of a horizontal plate impacting a flat liquid surface is investigated through velocity potential flow theory. The inner solution is matched with the outer solution. The far field of the inner solution is assumed to be far away from the other edge of the plate, and thus, its effect can be neglected. The effects of surface tension, viscous friction, and gravity are accounted for in the fully nonlinear dynamic boundary condition on the free surface. When one of these effects is dominant and the other two can be ignored, it is then possible to use self-similar variables to describe the local flow if the entry speed varies with time in a corresponding manner. Detailed results for various self-similar solutions are provided, and the relative importance of the Weber number, Reynolds number, and Froude number is investigated. Simulations are also undertaken for general non-similar flow, and the comparison with the experimental data is also made.
\end{abstract}

Published under license by AIP Publishing. https://doi.org/10.1063/5.0013914

\section{INTRODUCTION}

The flow near the plate edge at the initial stage during water entry has received considerable amount of interest. An accurate solution not only provides some insight into the complex flow features at this stage but also provides a solid foundation for the subsequent simulation for the flow at later stages, and all these have important practical applications. One example is a high speed craft in rough seas. Its cross section is often of V-shape, which tends to a horizontal plate when the deadrise angle is small. Persistent slamming may seriously damage its structure. Thus, understanding the physical feature of the flow at the plate edge is of high practical relevance. For irrotational flow, when a horizontal plate moves into water suddenly at time $t=0$, the velocity at the plate edge from the solution of the velocity potential is infinite. To remove the singularity, a local self-similar solution at the plate edge is often introduced for $t>0$.

Using self-similarity for local flow at the initial stage of impact has been adopted by several authors, usually based on the assumptions that viscosity and gravity are not important. Yakimov ${ }^{1}$ undertook an experimental study for flow near the corner of a plate impacting the free surface. He discussed that local flow could be selfsimilar and the spatial variables $x, y$ could be combined with time $t$ in the manner of $x /\left(c t^{2 / 3}\right), y /\left(c t^{2 / 3}\right)$. Iafrati and Korobkin ${ }^{2}$ used this principle and undertook detailed analysis through numerical solution for the local flow near the plate edge. The outer solution far away from the plate corner was obtained from the initial solution of the plate impacting the free surface at $t=0$. To the inner region, the form of the outer solution was the same as that of a semi-infinite plate. The inner solution of the edge was obtained through the selfsimilar variables when higher order terms of $t$ were ignored. To match the outer solution, the exponent $2 / 3$ was chosen for $t$, as in the work of Yakimov. ${ }^{1}$ They provided the free surface near the corner, including the jet, based on the inner solution. The result was quite similar to that measured by Yakimov. ${ }^{1}$ When the surface tension was included, the scaling of $2 / 3$ could still be used. However, Iafrati and Korobkin ${ }^{2}$ encountered some difficulties in numerical computation of surface tension and results for its effect were not provided. Later, Iafrati and Korobkin ${ }^{3}$ matched the inner solution with the higher order outer solution. Apart from the work mentioned above, the 2/3 scaling has also been used in some similar problems, for example, the jet formed during the collapse of the surface under the effect of inertial forces by Zeff et al., ${ }^{4}$ flow driven by surface tension by Keller, Milewski, and Vanden-Broeck, ${ }^{5}$ capillary pinch-off by Leppinen and Lister, ${ }^{6}$ the free surface deformation during the early time for the water entry of a small cylinder by Vella and $\mathrm{Li}^{\mathrm{y}}$, and the splash wave and crown breakup after the disk impact on a liquid surface by Peters, van der Meer, and Gordillo.

Krechetnikov ${ }^{9}$ suggested that the self-similar flow with $2 / 3$ scaling was not appropriate for studying the limit $t \rightarrow 0$ once the 
viscosity effect was taken into account. When such an effect is important, the inner solution could not be matched with the outer solution at such a scaling. Instead, the scaling of $1 / 2$ was proposed for the exponent of $t$ at the initial stage and the viscous effect could be more important than the others such as surface tension and gravity. Mayer and Krechetnikov ${ }^{10}$ undertook an experimental study on the local flow of a plate impacting on the water surface with moderate Reynolds and Weber numbers, to investigate the effects of viscosity and surface tension on the velocity field and ejecta evolutions. They found that their results did not match well with those from the work of Yakimov ${ }^{1}$ or Iafrati and Korobkin ${ }^{2}$ based on 2/3 scaling. Instead, the scaling of $3 / 4$ was found to match their experimental data best in some cases, as shown in Fig. 18 of their work, where $t$ is very small and the Weber number is much smaller than the Reynolds number.

In reality, for a particular physical problem of a plate impacting the free surface, the scaling of local flow will very much depend on the relative importance of various physical parameters such as gravity, surface tension, and viscosity and different stages of the flow. When a particular scaling related to a particular effect is dominant, it is then possible mathematically to use self-similar variables to describe the local flow when scaling related to the other effects is ignored. However, this scaling has to be also compatible with the outer flow in the mathematical model, which also depends on how the impact speed changes with time. In this work, we shall consider the local flow at the edge of a plate with length $2 L$ impacting the liquid surface with speed $V=V_{0}\left(t V_{0} / L\right)^{b}$. The local solution can be matched with an outer solution. The far field of the inner solution is assumed to be far away from the other edge of the plate, and thus, high order terms of the outer solution can be neglected. We shall discuss systematically when the self-similar solution would be possible and what corresponding scaling should be used in the formulation. Then, we shall consider the inner solution in the time domain, including the effects of surface tension, viscous friction on the free surface, and gravity. This is to show that it is not necessary to limit the inner solution to the self-similar one. The time domain solution can be used to resolve the singularity at the edge flow. In fact, it would be more convenient to connect the time domain solution with the solution at large $t$, where the solution will not be self-similar.

In Sec. II, we shall first outline the mathematical model of the plate impacting the free surface. The governing equation and boundary conditions are introduced based on the velocity potential theory. The matching between the local solution and outer solution, the choice of scaling, and conditions for a self-similar solution are discussed. In Sec. III, self-similar solutions are first considered and results are provided. This is followed by time domain solutions, where the comparisons with experimental data are made and extensive analysis is made.

\section{MATHEMATICAL MODEL}

Figure 1 shows a plate with length $2 L$ initially floating on the calm free surface. A Cartesian coordinate system $O-x y$ is defined so that the $x$-axis coincides with the undisturbed free surface and the $y$-axis points upward and passes the right edge of the plate. It is assumed that the fluid is inviscid and incompressible, and the fluid

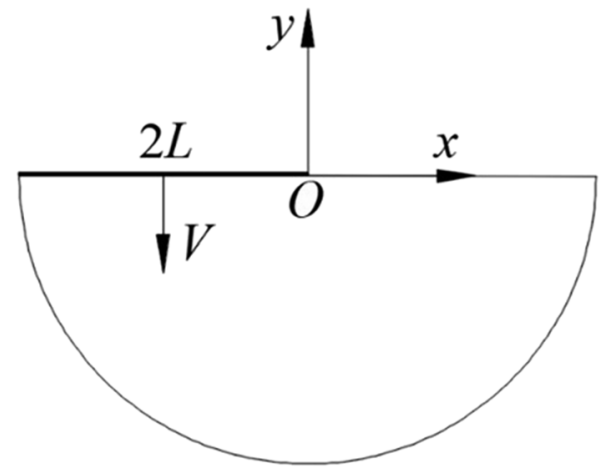

FIG. 1. The sketch of the problem.

motion can be described with potential $\phi$, whose gradient is equal to fluid velocity or $v=\nabla \phi$. The plate moves down suddenly with velocity $V$. If we focus on the local flow near the edge of the plate, the solution for the velocity potential flow at $t=0$ can be written as ${ }^{9}$

$$
\phi=\Phi=\operatorname{Re}[i V(z-\sqrt{2 z L})]
$$

where $z=x+y i$ and $\Phi(x, y)=\phi(x, y, t=0)$ is the initial solution, which is in fact the same as the steady-state solution of a uniform flow around a plate in an unbounded fluid domain. This solution gives a singularity of the velocity at $z=0$. Therefore, an inner solution is needed near the region of $z \cong 0$.

When $t>0$, the governing equation and boundary conditions for $\phi$ for the inner solution can be written as

$$
\begin{gathered}
\Delta \phi=0 \text { in the fluid domain, } \\
\phi_{y}=-V \quad(x<0, y=-h), \\
\partial \zeta / \partial t=\phi_{y}-\zeta_{x} \phi_{x} \quad(y=\zeta(x, t), x>0), \\
\partial \phi / \partial t=-\frac{1}{2}\left(\phi_{x}^{2}+\phi_{y}^{2}\right)+\frac{\sigma \kappa}{\rho}+2 v \lambda-g y \quad(y=\zeta(x, t), x>0),
\end{gathered}
$$

where $\zeta$ is the elevation of the free surface, $h=\int_{0}^{t} V d \tau$ is the vertical distance that the plate has traveled, $\sigma$ is the surface tension coefficient, $\rho$ is the fluid density, $v$ is the kinematic viscosity, and $g$ is the acceleration due to gravity. In Eq. (5), $\kappa=x_{l_{0}} y_{l_{0} l_{0}}-y_{l_{0}} x_{l_{0} l_{0}}$ is the curvature, ${ }^{5}$ where subscript $l_{0}$ indicates the arc length coordinate along the free surface and ${ }^{11} \lambda=\left[\frac{\partial u_{x}}{\partial x} n_{x}^{2}+\left(\frac{\partial u_{x}}{\partial y}+\frac{\partial u_{y}}{\partial x}\right) n_{x} n_{y}+\frac{\partial u_{y}}{\partial y} n_{y}^{2}\right]$ is due to the contribution of the shear force along the surface perpendicular to the free surface, to the balance of force with pressure on the free surface, with $\nabla \phi=\left(u_{x}, u_{y}\right)$ and $\mathbf{n}=\left(n_{x}, n_{y}\right)$ being the normal of the free surface. Here, we have included the shear force effect under the free surface boundary condition but have ignored the viscous effect in the fluid domain. At $t=0$, the initial condition can be written as

$$
\begin{gathered}
\zeta(x, t=0)=0, \\
\phi(x, y, t=0)=\psi(x, y) .
\end{gathered}
$$


We may define that $(\tilde{x}, \tilde{y})$ moves with the plate and then introduces the following transformation:

$$
\begin{gathered}
x=\tilde{x}, y=\tilde{y}-h, \zeta=\tilde{\zeta}-h, \\
\phi=\tilde{\phi}-V \tilde{y}+\frac{1}{2} \int_{0}^{t} V^{2}(\tau) d \tau+g \int_{0}^{t} h(\tau) d \tau .
\end{gathered}
$$

The governing equation and boundary conditions can be written as

$$
\begin{gathered}
\Delta \tilde{\phi}=0 \text { in the fluid domain, } \\
\tilde{\phi}_{\tilde{y}}=0 \quad(\tilde{x}<0, \tilde{y}=0), \\
\partial \tilde{\zeta} / \partial t=\tilde{\phi}_{\tilde{y}}-\tilde{\zeta}_{\tilde{x}} \tilde{\phi}_{\tilde{x}} \quad(\tilde{y}=\tilde{\zeta}(\tilde{x}, t), \tilde{x}>0), \\
\frac{\partial \tilde{\phi}}{\partial t}=-\frac{1}{2}\left(\tilde{\phi}_{\tilde{x}}^{2}+\tilde{\phi}_{\tilde{y}}^{2}\right)+\dot{V} \tilde{y}+\frac{\sigma \kappa}{\rho}-g \tilde{y}+2 v \lambda \quad(\tilde{y}=\tilde{\zeta}(\tilde{x}, t), \tilde{x}>0) .
\end{gathered}
$$

Correspondingly, the outer solution in Eq. (1) can be written as

$$
\tilde{\phi}=\tilde{\psi}=-V \sqrt{L} \operatorname{Re}(i \sqrt{2 \tilde{z}}),
$$

where $\tilde{z}=\tilde{x}+\tilde{y} i$. The additive spatial independent constant is ignored in Eq. (13). The solution of Eqs. (9)-(12) should match the outer solution away from the edge. Strictly speaking, Eq. (13) is only for $t=0$ or $h=0$. For $h \neq 0$, we may follow Iafrati and Korobkin ${ }^{3}$ and expand the outer solution in terms of $h$. Using their $w_{0}$ and $w_{1}$, we have near $\tilde{z}=0$ the following:

$$
\tilde{\phi}=V L \operatorname{Re}[-i \sqrt{2 \tilde{z} / L}-(h / L) /(2 \tilde{z} / L)+2 i s C / \sqrt{2 \tilde{z} / L}],
$$

where $C$ is a constant. The inner solution may be written in terms of the local coordinate system. We may write

$$
\tilde{\phi}(\tilde{x}, \tilde{y}, t)=L V \sqrt{2 s} \varphi(\alpha, \beta, t), \tilde{x}=L s \alpha, \tilde{y}=L s \beta, \tilde{\zeta}=L s \eta .
$$

Let $V_{0}$ be the characteristic velocity. We may assume that the velocity $V=V_{0}\left(t V_{0} / L\right)^{b}$ and take $s=A\left(t V_{0} / L\right)^{a}$. Substituting Eq. (15) into (9)-(12), and replacing $t$ with $s$ for the temporal variation, we have

$$
\begin{gathered}
\Delta \varphi=0 \text { in the fluid domain, } \\
\varphi_{\beta}=0 \quad(\alpha<0, \beta=0) \\
\frac{A^{\frac{b+1}{a}} a}{\sqrt{2}} \frac{s^{\frac{3}{2}-\frac{b+1}{a}} \partial s \eta}{\partial s}=\left(\varphi_{\beta}-\eta_{\alpha} \varphi_{\alpha}\right) \quad(\beta=\eta(\alpha, s), \alpha>0) \\
\frac{A^{\frac{b+1}{a}} a}{\sqrt{2}} \frac{\partial s^{\frac{1}{2}+\frac{b}{a}} \varphi}{s^{\frac{2 b-2 a+1}{a}} \partial s}=-\frac{1}{2}\left(\varphi_{\alpha}^{2}+\varphi_{\beta}^{2}\right)+\frac{b}{2} A^{\frac{b+1}{a}} s^{2-\frac{b+1}{a}} \beta+\frac{A^{2 c} s^{-2 c} \bar{\kappa}}{2 W_{e}^{2}} \\
+\frac{\sqrt{2} A^{c} s^{-c-\frac{1}{2}} \bar{\lambda}}{R_{e}}-\frac{A^{2 c} s^{2-2 c} \beta}{2 F_{r}^{2}}(\beta=\eta(\alpha, s), \alpha>0)
\end{gathered}
$$

where $c=b / a, W_{e}=V_{0} \sqrt{\rho L / \sigma}$ is the Weber number, $\bar{\kappa}=\alpha_{l} \beta_{l l}-\beta_{l} \alpha_{l l}$ is the nondimensionalized curvature, $R_{e}=V_{0} L / v$ is the Reynolds number, $\bar{\lambda}=\varphi_{l l}-\beta_{l l} \varphi_{\beta}-\alpha_{l l} \varphi_{\alpha}$, and $F_{r}=V_{0} / \sqrt{g L}$ is the Froude number. The initial free surface elevation is assumed to be zero, or

$$
\eta(\alpha, 0)=0
$$

together with the potential on the free surface,

$$
\varphi(\alpha, 0,0)=0 .
$$

The far field boundary condition for the inner solution takes the form as

$$
\varphi=\sqrt{r} \sin (\theta / 2)-h \cos \theta /\left(L 2 \sqrt{2} s^{3 / 2} r\right)+C \sin (\theta / 2) / \sqrt{r}, \quad r \rightarrow \infty,
$$

where $r=\sqrt{\alpha^{2}+\beta^{2}}$. The first term will be the leading term, provided $h / s^{3 / 2}$ is finite as $t \rightarrow 0$ or when $a \leq 2(b+1) / 3$.

In the case of a self-similar solution, we must have $\frac{\partial \eta}{\partial s}=0$ and $\frac{\partial \varphi}{\partial s}=0$. For this to be possible, it is also necessary to have $a=\frac{2}{3}(b+1)$ in (18) and (19), which meets the above condition for $a$. We may also let $A=(\sqrt{2} / a)^{2 / 3}$, and then, Eqs. (18) and (19) become

$$
\begin{aligned}
\frac{\partial s \eta}{\partial s}= & \left(\varphi_{\beta}-\eta_{\alpha} \varphi_{\alpha}\right) \quad(\beta=\eta(\alpha, s), \alpha>0), \\
\frac{\partial s^{2-\frac{1}{a}} \varphi}{s^{1-\frac{1}{a}} \partial s}= & -\frac{1}{2}\left(\varphi_{\alpha}^{2}+\varphi_{\beta}^{2}\right)+\frac{c \sqrt{s} \beta}{\sqrt{2}}+\left(\frac{\sqrt{2}}{a}\right)^{\frac{4 c}{3}} \frac{s^{-2 c} \bar{\kappa}}{2 W_{e}^{2}} \\
& +\left(\frac{\sqrt{2}}{a}\right)^{\frac{2 c}{3}} \frac{\sqrt{2} s^{-c-\frac{1}{2}} \bar{\lambda}}{R_{e}} \\
& -\left(\frac{\sqrt{2}}{a}\right)^{\frac{4 c}{3}} \frac{s^{2-2 c} \beta}{2 F_{r}^{2}} \quad(\beta=\eta(\alpha, s), \alpha>0) .
\end{aligned}
$$

In such a case, $h=L(s / A)^{3 / 2} /(b+1)$ and only the first term in Eq. (22) needs to be kept. The far field boundary condition becomes

$$
\varphi=\sqrt{r} \sin (\theta / 2), \quad r \rightarrow \infty
$$

or

$$
\varphi_{r}=\frac{1}{2 \sqrt{r}} \sin \frac{\theta}{2}, \quad r \rightarrow \infty,
$$

and the latter is used in the numerical solution procedure.

In Eq. (24), the last three terms are due to the effects of surface tension, viscosity, and gravity, respectively. If these effects are all neglected, or $W_{e}, R_{e}$, and $F_{r}$ all tend to infinity, at an early stage, the solution may be self-similar when the remaining higher order term $\frac{c \sqrt{s} \beta}{\sqrt{2}}$ is ignored if $s$ is small. In such a case, $\eta_{s}$ and $\varphi_{s}$ terms can be taken as zero and Eqs. (23) and (24) then take the form of

$$
\begin{gathered}
\eta-\alpha \eta_{\alpha}=\varphi_{\beta}-\eta_{\alpha} \varphi_{\alpha}, \quad \beta=\eta(\alpha), \\
\left(2-\frac{1}{a}\right) \varphi-\alpha \varphi_{\alpha}-\beta \varphi_{\beta}=-\frac{1}{2}\left(\varphi_{\alpha}^{2}+\varphi_{\beta}^{2}\right), \quad \beta=\eta(\alpha),
\end{gathered}
$$

which is valid for any $a$.

In general, if all the terms of $W_{e}, R_{e}$, and $F_{r}$ are important, the self-similar solution is not possible even at small $s$. The relative importance of each term will depend on their values as well as $a$ and $s$. When only one of these effects is important and the other two can be ignored, a self-similar solution would still be possible. For example, when the surface tension is included and viscous and gravity effects are ignored at $b=0$ and $a=2 / 3$, Eq. (24) takes the form of

$$
\frac{1}{2} \varphi-\alpha \varphi_{\alpha}-\beta \varphi_{\beta}=-\frac{1}{2}\left(\varphi_{\alpha}^{2}+\varphi_{\beta}^{2}\right)+\frac{\bar{\kappa}}{2 W_{e}^{2}}, \quad \beta=\eta(\alpha) \text {. }
$$


Equations (27) and (29) are the same as Eqs. (18) and (19) in the work of Iafrati and Korobkin. ${ }^{2}$ However, their derivation is done in the coordinate system $O-x y$. Then, assumptions are made through which the first term in Eq. (1) and higher order terms of $t$ in the dynamic free surface condition are ignored. Here, we have derived equations based on the moving system $O-\tilde{x} \tilde{y}$ and the transformation in Eq. (8), and therefore, no such assumptions are made.

If the $R_{e}$ effect is included, and $W_{e}$ and $F_{r}$ effects can be ignored, at $a=1 / 2$, Eq. (24) becomes

$$
-\alpha \varphi_{\alpha}-\beta \varphi_{\beta}=-\frac{1}{2}\left(\varphi_{\alpha}^{2}+\varphi_{\beta}^{2}\right)+\frac{\bar{\lambda}}{R_{e}}, \quad \beta=\eta(\alpha)
$$

after the $\sqrt{s}$ term is ignored at small $s$. If the $F_{r}$ effect is included, and $W_{e}$ and $R_{e}$ effects can be ignored, at $a=2$, Eq. (24) then becomes

$$
\frac{3}{2} \varphi-\alpha \varphi_{\alpha}-\beta \varphi_{\beta}=-\frac{1}{2}\left(\varphi_{\alpha}^{2}+\varphi_{\beta}^{2}\right)-\frac{\beta}{2^{5 / 3} F_{r}^{2}}, \quad \beta=\eta(\alpha)
$$

after the $\sqrt{s}$ term is ignored.

We may consider more carefully the flow condition at the corner. Here, we may assume that both the pressure and velocity are continuous, similar to the Kutta condition at the trailing edge of an aero/hydrofoil. In such a case, the relative velocity of the flow leaving the plate edge should be tangential to the plate. In the case of a self-similar flow, Eq. (27) is always valid. We may apply Eqs. (17)(27) at $\alpha=0$ and $\beta=0$. This gives $\eta_{\alpha} \varphi_{\alpha}=0$, which means that either $\eta_{\alpha}=0$ or $\varphi_{\alpha}=0$. Without the Kutta condition, the fluid can leave the plate surface in an arbitrary direction and singularity may appear in the velocity and pressure.

\section{NUMERICAL RESULTS AND DISCUSSIONS}

The problem is solved using the time stepping method through $s$ based on the Lagrangian form of the free surface boundary conditions. As the matching condition with the far field has imposed $a \leq 2(b+1) / 3$, we may consider the case $a=2(b+1) / 3$ with $\sqrt{2}=A^{3 / 2} a$. Then, we have

$$
\begin{gathered}
\frac{D(s \alpha)}{D s}=\varphi_{\alpha}, \frac{D(s \beta)}{D s}=\varphi_{\beta}, \\
\frac{D s \varphi}{D s}=\frac{1}{2}\left(\varphi_{\alpha}^{2}+\varphi_{\beta}^{2}\right)-\left(1-\frac{1}{a}\right) \varphi+\frac{c \sqrt{s} \beta}{\sqrt{2}}+\left(\frac{\sqrt{2}}{a}\right)^{\frac{4 c}{3}} \frac{s^{-2 c} \bar{\kappa}}{2 W_{e}^{2}} \\
+\left(\frac{\sqrt{2}}{a}\right)^{\frac{2 c}{3}} \frac{\sqrt{2} s^{-c-\frac{1}{2}} \bar{\lambda}}{R_{e}}-\left(\frac{\sqrt{2}}{a}\right)^{\frac{4 c}{3}} \frac{s^{2-2 c} \beta}{2 F_{r}^{2}}
\end{gathered}
$$

Here, $\frac{d s^{2-\frac{1}{a}} \varphi}{s^{1-\frac{1}{a}} d s}=\left(1-\frac{1}{a}\right) \varphi+\frac{d s \varphi}{d s}$ has been used in Eq. (33). The solution starts from $s=0$. If only one of the last three terms on the righthand side of (33) is retained with the corresponding $a$, neglecting the term $\frac{c \sqrt{s} \beta}{\sqrt{2}}$ in Eq. (33), after a period of transition, the solution will become self-similar, or it no longer changes with $s$. In practical solution computations, $s$ will start from an extremely small $s_{0}$. Then, as $s / s_{0}$ increases, the solution tends to be self-similar. In fact, as observed by Wu and $\mathrm{Sun}^{12}$ in a related problem, it is already sufficient when $s / s_{0} \geq 10$. At each step of $s$, the Laplace equation is solved based on the boundary element method used in the work of Sun et al., ${ }^{13}$ which is also used by Wang, Faltinsen, and Lugni ${ }^{14}$ for a similar water entry problem, and the fluid particle is assumed to leave the plate tangentially because its normal velocity is the same as that of the plate. When there is a very thin jet, it is treated by the method in the work of $\mathrm{Bao}, \mathrm{Wu}$, and $\mathrm{Xu},{ }^{15}$ taking into account the surface tension and viscosity effects on the free surface.

\section{A. Convergence study and comparison}

In order to test the numerical stability and accuracy of the methodology, we first consider the case with the infinite Weber number, Reynolds number, and Froude number, and $a=2 / 3$ and $b=0$. The half width and depth of the rectangular computational domain $L_{d}$ are both set as 50 in the $O-\alpha \beta$ system. The calculation starts with a small initial value $s_{0}=0.001$ and advances through $d s$. The step $d s$ varies with time. It starts from a smallest step $d s_{m}$, then increases at a fixed ratio $\delta_{s}$, and is not allowed to exceed an upper $d s_{L}$. Unequal elements are distributed along the fluid boundary. The smallest elements of length $l_{\mathrm{m}}$ are used at the edge of plate; away from the edge, the size of the element increases gradually at a fixed ratio $\delta_{l}$, but is limited by a maximum of 0.5 . For the mesh convergence study, we set $l_{m}$ as $0.01,0.02$, and 0.03 and $\delta_{l}$ as 1.02 . $d s_{m}, \delta_{s}$, and $d s_{L}$, for the time step is set as $5 \times 10^{-7}, 1.0005$, and $5 \times 10^{-5}$, respectively. The free surface profiles with these three different meshes are shown in Fig. 2(a). They are taken when the profiles become steady in the self-similar coordinate system. A good agreement can be seen in this figure, which means that the results are mesh independent. The comparison of results from two different time steps is shown in Fig. 2(b) to verify the convergence with the time step, with $l_{m}=0.02$. The time step in the first case is the same as that in the mesh convergence study. In the second case, the corresponding time steps are reduced by half, or $d s_{m}=2.5 \times 10^{-7}$, $\delta_{s}=1.00025$, and $d s_{L}=2.5 \times 10^{-5}$. It can be seen that the two curves do not have a visible difference, which shows that the results have converged with the time step. To test the convergence of the size of the computational domain, $L_{d}$ is increased to 200 and good agreement can be seen in Fig. 2(c). The comparison between the present result and that by Iafrati and Korobkin ${ }^{2}$ is given in Fig. 2(d). The good agreement means that the present procedure is accurate.

\section{B. Similarity solutions}

\section{Results at different a with $W_{e}=F_{r}=R_{e} \rightarrow \infty$}

Figure 3 gives the similarity solutions at different $a$ with infinite Weber numbers, Reynolds numbers, and Froude numbers. This means that the surface tension, viscosity effect, and gravity effect are all neglected. If the remaining $\beta$ term in the dynamic boundary condition is also neglected, $a$ can be arbitrarily chosen and the solution can be self-similar. As $a$ increases, $b$ also increases, based on the relation of $a=\frac{2}{3}(b+1) \cdot a=2 / 3$ corresponds to $b=0$ or the impact at constant speed, while $a<2 / 3$ corresponds to the decelerating impact and $a>2 / 3$ corresponds to the accelerating impact. In Fig. 3 , it can be seen that the free surface jet points rightward at smaller $a$. This is because when $a<2 / 3$ or $b<0$, the impact speed at $t=0$ is infinite. The fluid below the plate will be ejected out with high speed. When $0<b<1$, or $2 / 3<a<4 / 3$, the plate starts motion with zero speed but infinite acceleration. The jet ejection from the plate becomes weaker, 

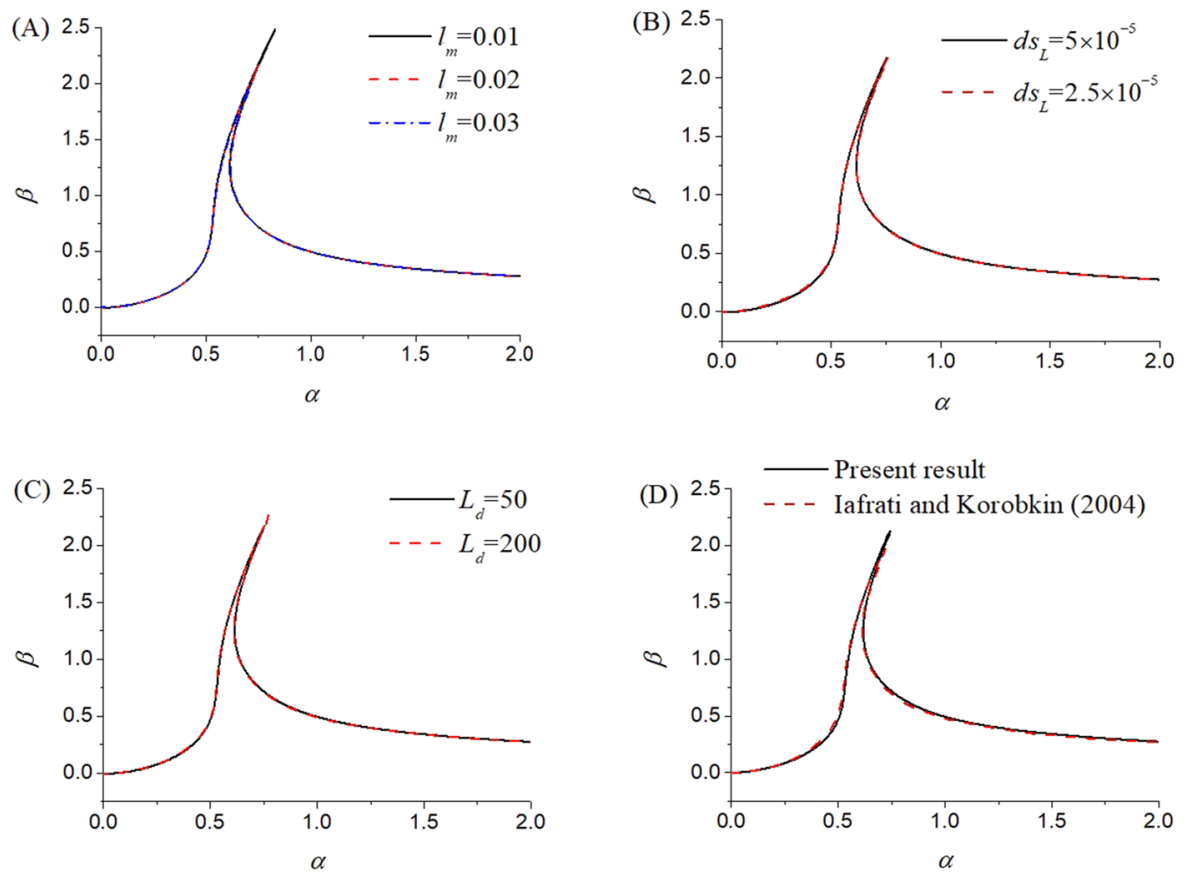

FIG. 2. Convergence study of (a) mesh $\left(d s_{L}=5 \times 10^{-5}\right.$ and $\left.L_{d}=50\right)$, (b) time step $\left(I_{m}=0.02\right.$ and $\left.L_{d}=50\right)$, (c) the size of the domain $\left(I_{m}=0.02\right.$ and $d s_{L}=5$ $\left.\times 10^{-5}\right)$, and (d) the comparison with the work of lafrati and Korobkin ${ }^{2}\left(I_{m}=0.02\right.$, $d s_{L}=5 \times 10^{-5}$, and $\left.L_{d}=50\right)\left(W_{e} \rightarrow \infty\right.$ $R_{e} \rightarrow \infty$, and $\left.F_{r} \rightarrow \infty\right)$. and the ejected jet gradually bends to the left. When $b>1$ or $a>4 / 3$, the motion of the plate starts with both zero speed and zero acceleration. As the plate moves down slowly, the fluid is more ready to move inward or to take the space left by the plate. In fact, at $a \geq 1.5$, the free surface begins to overturn.

\section{Results with finite Weber, Reynolds, or Froude numbers}

With the scale of $2 / 3$, the free surfaces at different Weber numbers with $R_{e}=F_{r}=\infty$ are provided in Fig. 4(a). When $W_{e} \geq 20$, the curves are no longer notably affected by $W_{e}$. As $W_{e}$ decreases, its most notable effect is near the jet tip, where the free surface has the

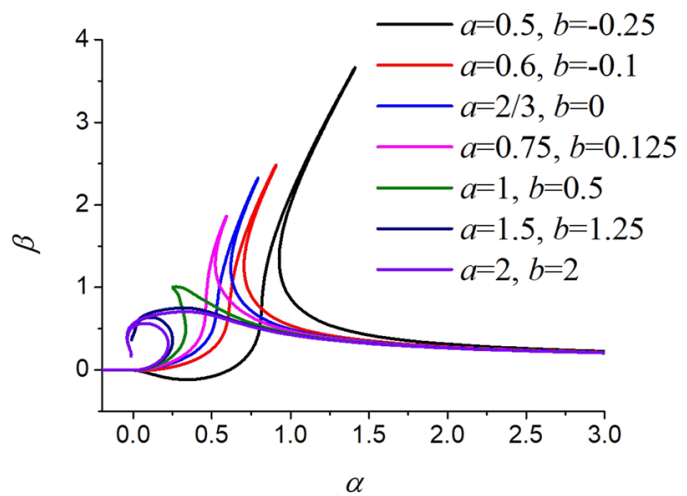

FIG. 3. Similarity solutions at different $a\left(W_{e} \rightarrow \infty, R_{e} \rightarrow \infty\right.$, and $\left.F_{r} \rightarrow \infty\right)$. largest curvature. The main free surface is not notably affected. As the Weber number continues to drop to 4 , its effects on the other parts of the free surface also become significant. A crown becomes visible near the jet tip, which is similar to that in the work of Peters et $a l^{8}$ At $W_{e}=2$, the jet has disappeared and the free surface becomes much flatter. In fact, as $W_{e} \rightarrow 0$, the dynamic condition means that the curvature of the free surface should be zero, and therefore, it will tend to be a flat surface.

Results at different Reynolds numbers $R_{e}$ and $a=1 / 2$ with $W_{e}=F_{r}=\infty$ are provided in Fig. $4(\mathrm{~b})$. The results are not very much affected by $R_{e}$ when $R_{e} \geq 200$. It is of interest to see that at larger $R_{e}$, the jet bends to the right side. When $R_{e}$ decreases, the jet gradually bends to the left. However, it should be noted that in the present simulations, the effect of the viscosity is accounted for only in the friction on the free surface and is ignored inside the fluid. This approximation may not be justified at low $R_{e}$.

The results at different Froude numbers $F_{r}$ with $a=2$ and $W_{e}=R_{e}=\infty$ are provided in Fig. 4(c). As $b=2$ in this case, motion starts with both zero velocity and acceleration. As discussed in Fig. 3, with $F_{r}=\infty$, the fluid in such a case moves inward to take the space left behind the plate. Figure 4(c) shows when $F_{r} \geq 5$ that the Froude number does not have a major effect and the result is close to that at $F_{r} \rightarrow \infty$. When $F_{r}=2$, the gravity effect on the main part of the free surface is still small. Its main effect is to pull the jet further down. As $F_{r}$ further drops, the jet tip will touch the plate and a closed cavity would therefore form, similar to what has been observed in the work of Semenov, $\mathrm{Wu}$, and Korobkin, ${ }^{16}$ which is beyond the scope of the current work. It should be pointed out that the effect of the gravity is considered in the particular case $a=b=2$ with which the flow may be approximated as self-similar. In other cases, the effect of gravity may be different. 

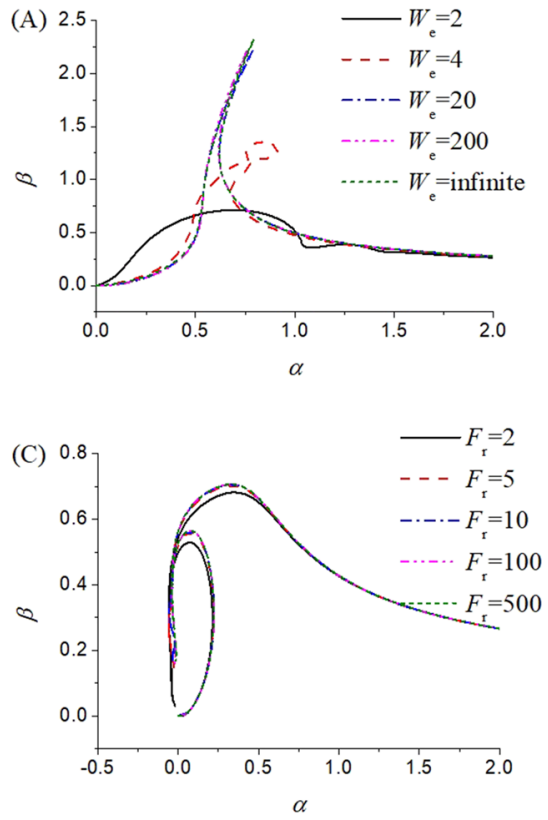

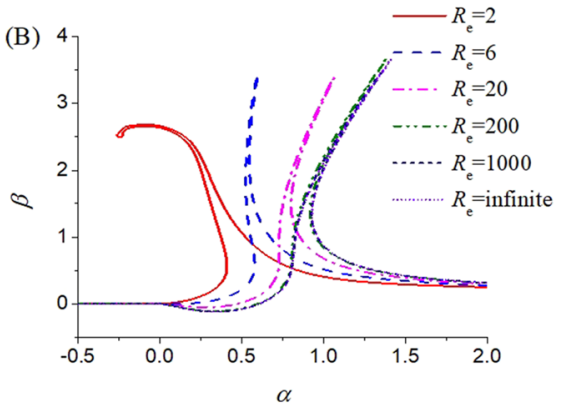

FIG. 4. Free surfaces at different (a) Weber numbers $\left(R_{e} \rightarrow \infty, F_{r} \rightarrow \infty\right.$ $a=2 / 3$, and $b=0)$, (b) Reynolds numbers $\left(W_{e} \rightarrow \infty, F_{r} \rightarrow \infty, a=1 / 2\right.$, and $b=-1 / 4)$, and (c) Froude numbers $\left(R_{e}\right.$ $\rightarrow \infty, W_{e} \rightarrow \infty, a=2$, and $b=2$ )

\section{Time domain solutions}

In general, the flow may not be self-similar. In the following case, the simulation starts at a very small $s_{0}$ or very small initial time step. Unlike the cases in Sec. III B, where the results were taken when the solution becomes self-similar, the results here are taken at the real time instants. When terms of $W_{e}, R_{e}$, and $F_{r}$ are included in the dynamic free surface boundary condition in Eq. (33), their importance will be dependent on their relative values, the values of $b$ and $t$. We may focus on the case of $b \geq 0$ with $a=2(b+1) / 3$ in which the entry speed will not be infinite at $t=0$. In such a case, the $W_{e}$ and $R_{e}$ terms may be important at small $s$ because their exponents are negative. When $0 \leq b<1 / 2$, which gives $0 \leq c<1 / 2$, the $R_{e}$ term can be more important than the $W_{e}$ term. This includes the case of constant speed water entry with $b=0$. The effect of the $F_{r}$ term is small at small $s$ because its exponent is positive. When $b>1 / 2$, or $c>1 / 2$, the $W_{e}$ term becomes more important than the $R_{e}$ term at small $s$. The $F_{r}$ term will never be more important than the $W_{e}$ term at small $s$, in terms of the exponents. The $F_{r}$ term may be more important at small $s$ when $b<-5 / 2$ or $c>5 / 2$. However, this is the case of infinite initial speed and even infinite initial $h$ and, therefore, is not considered here.

\section{The comparison with the experiment}

We consider a case in an experiment undertaken by Mayer and Krechetnikov $^{10}$ for a plate falling into water with half width $L=25 \mathrm{~mm}$ and a constant vertical velocity $V_{0}=0.25 \mathrm{~m} / \mathrm{s}$. As in Fig. 18(a) of their work, we take the surface tension coefficient as $\sigma=0.072 \mathrm{~N} / \mathrm{m}^{2}$, water density as $\rho=999 \mathrm{~kg} / \mathrm{m}^{3}$, the dynamic viscosity coefficient as $\mu=1 \mathrm{mPa} \mathrm{s}$, and the acceleration of gravity as $g=9.8 \mathrm{~m} / \mathrm{s}^{2}$. All these give $W_{e}=4.658, R_{e}=6250$, and $F_{r}=0.505$.
Strictly speaking, as the experiment was undertaken with constant speed, we should have $b=0$ and $a=2 / 3$. Mayer and Krechetnikov ${ }^{10}$ plotted measured free surfaces in terms of $\alpha$ and $\beta$ at different time instants and found that these curves did not coincide very well. This suggested that the experimental data did not follow well the selfsimilar solution with $a=2 / 3$ and $b=0$. They then found that when they plotted the experimental data in terms of $\alpha$ and $\beta$ with $a=3 / 4$, all the curves were most close to each other. This suggested that the self-similar solution for $a=3 / 4$ might fit the experimental data best. However, in such a case, the requirement $a=2(b+1) / 3$ for a selfsimilar solution means that $b=1 / 8$, which is not constant speed. Nevertheless, $b$ is relatively small. Here, we have run simulations with different $a$, including $a=2 / 3$ and $3 / 4$, and found that $a=0.71$ $(b=0.065)$ fitted the experimental data best, as shown in Fig. 5 in which $\tau=L / V_{0}$. It should be emphasized that when all the terms in Eq. (33) are retained, the solution is not self-similar and the comparison is, therefore, made in the time domain. It should also be noted that constant $V_{0}$ was maintained in the experiment. However, it can also be noted that when the servo motor in the experiment drove the plate from air to denser water, more power would be needed to balance the increased resistance, and then, the plate might experience a short period of acceleration to reach the constant velocity $V_{0}$. This indicates that there might be a short period of time with varying $b$. In the present simulation, $V$ varies from $t=0$ to $t=\tau$, when $b \neq 0$ and $V_{0}$ is the speed at $t=\tau$. This figure shows that the results are very sensible to $b$. A small variation from $b=0$ to $b=1 / 8$ makes a major difference to the numerical result and its comparison with the measured data.

In another experiment shown in Fig. 19(a) of the work of Mayer and Krechetnikov, ${ }^{10}$ the dynamic viscosity coefficients are chosen as $\mu=1 \mathrm{mPa} \mathrm{s}, 100 \mathrm{mPa} \mathrm{s}$, and $1000 \mathrm{mPa}$ s, respectively. In the experiment, $\mu=1 \mathrm{mPa}$ s is combined with $\rho=999 \mathrm{~kg} \mathrm{~m}^{-3}$ and 

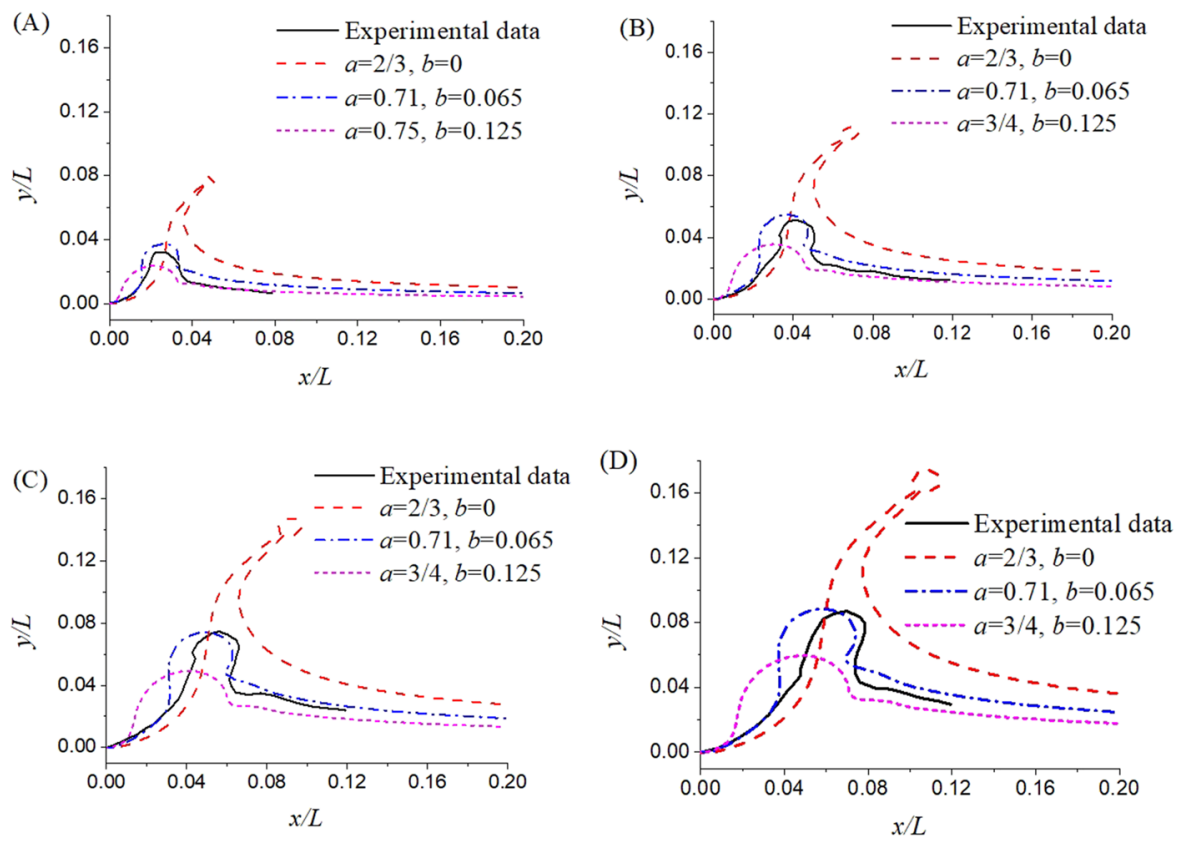

FIG. 5. The comparison of the free surface shape (dashed lines) with the experimental data (solid lines) by Mayer and Krechetnikov ${ }^{10}\left(W_{e}=4.658, R_{e}=6250\right.$, and $F_{r}=0.505$ ): (a) $t / \tau=0.006$, (b) $t / \tau=0.01$, (c) $t / \tau=0.015$, and (d) $t / \tau=0.019$.

$\sigma=0.072 \mathrm{~N} \mathrm{~m}^{-1}, \mu=100 \mathrm{mPa}$ s is combined with $\rho=1150 \mathrm{~kg} \mathrm{~m}^{-3}$ and $\sigma=0.065 \mathrm{~N} \mathrm{~m}^{-1}$, and $\mu=1000 \mathrm{mPa} \mathrm{s}$ is combined with $\rho=1250 \mathrm{~kg} \mathrm{~m}^{-3}$ and $\sigma=0.063 \mathrm{~N} \mathrm{~m}^{-1}$. The constant speed is set as $V_{0}=0.28 \mathrm{~m} / \mathrm{s}$, while the rest of the parameters are kept the same as those in Fig. 5. In such a case, we have three Weber numbers $W_{e}=5.217,5.491$, and 5.578, three Reynolds numbers $R_{e}=6993$,
80.5, and 8.75, and the same Froude number $F_{r}=0.565$. As stated by Mayer and Krechetnikov, ${ }^{10}$ the variation of the Weber number in this case is small. The difference in the results is mainly caused by the change in Reynolds numbers. Figure 6 gives the comparison between the present results at $a=0.71$ and the experimental data shown in Fig. 19(a) of the work of Mayer and Krechetnikov. ${ }^{10}$
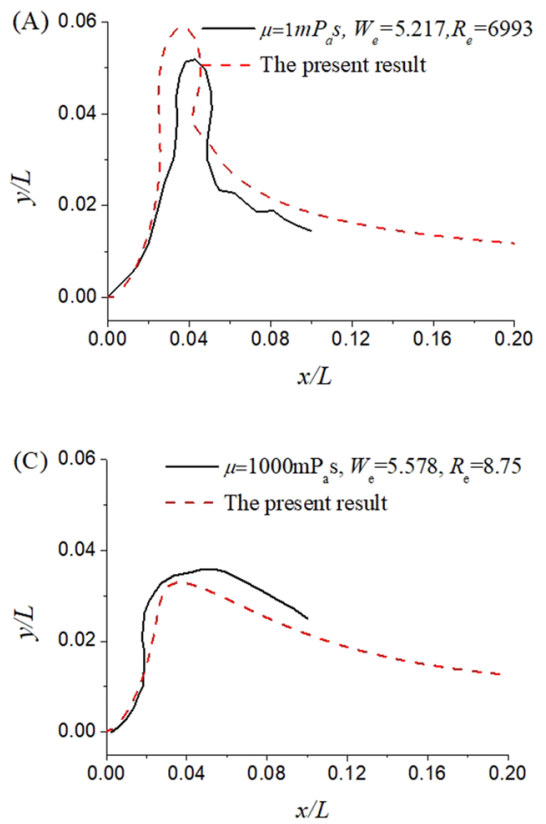

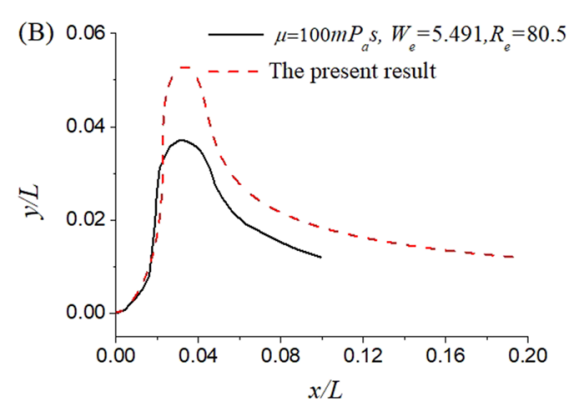

FIG. 6. The comparison of the free surface shape with the experimental data by Mayer and Krechetnikov ${ }^{10}$ at different Reynolds numbers $(a=0.71, b=0.065$, and $\left.F_{r}=0.565\right)$. 

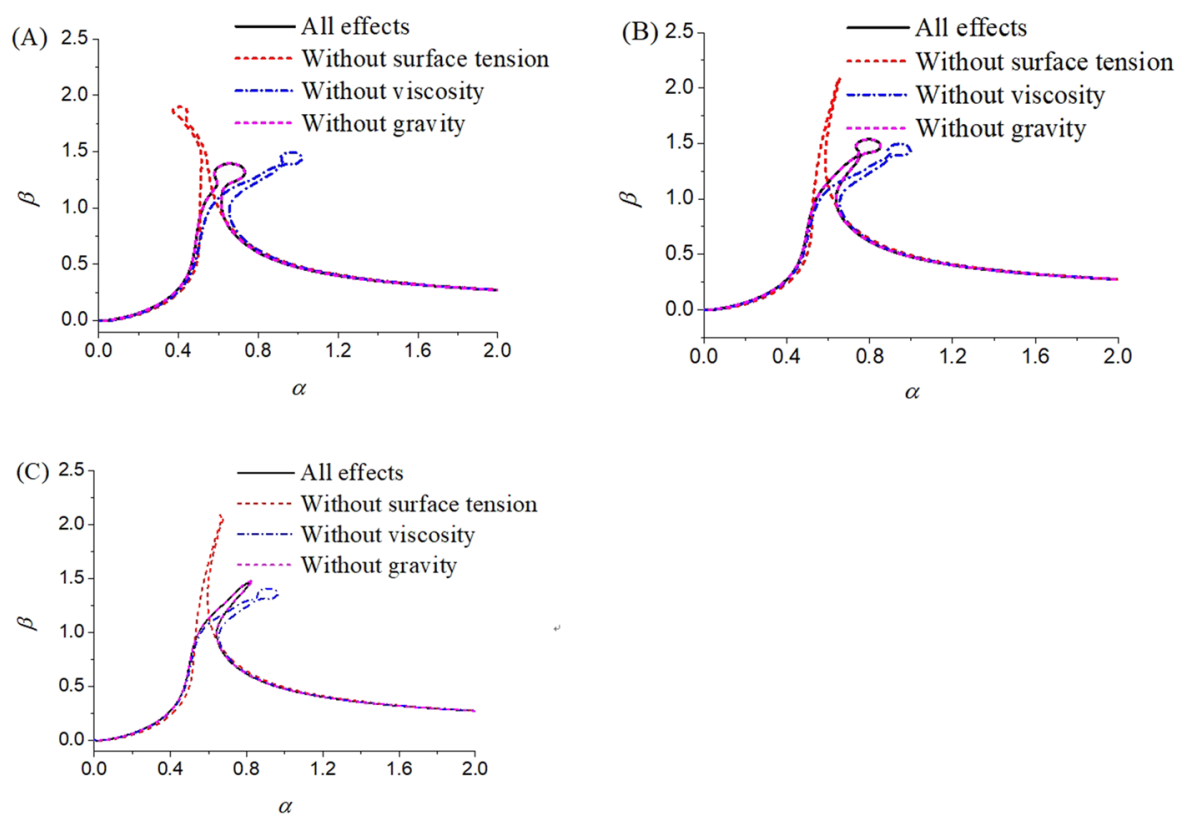

FIG. 7. The results for $(a=2 / 3, b=0)$ at different entry distances: (a) $s=0.01$ and $h / L=0.00471$, (b) $s=0.05$ and $h / L=0.00521$, and $(c) s=0.1$ and $h / L=0.0149$.
The snapshots of the free surface are all taken at $t / \tau=0.01$. It can be seen that the present result is in better agreement with the experimental data at the higher Reynolds number or $R_{e}=6993$. At $R_{e}=80.5$, the shape of the free surface is similar to that from the experiment. However, the free surface at the peak and beyond the peak is higher than that from the experiment. At $R_{e}=8.75$, the peak value of the free surface elevation is close to the experimental data, but the location is slightly different. Beyond the peak, the present result is lower than the experimental data. As discussed previously, in the present work, the viscous effect is accounted for only in the friction on the free surface and is ignored inside fluid. It is a major simplification at small Reynolds numbers. However, the
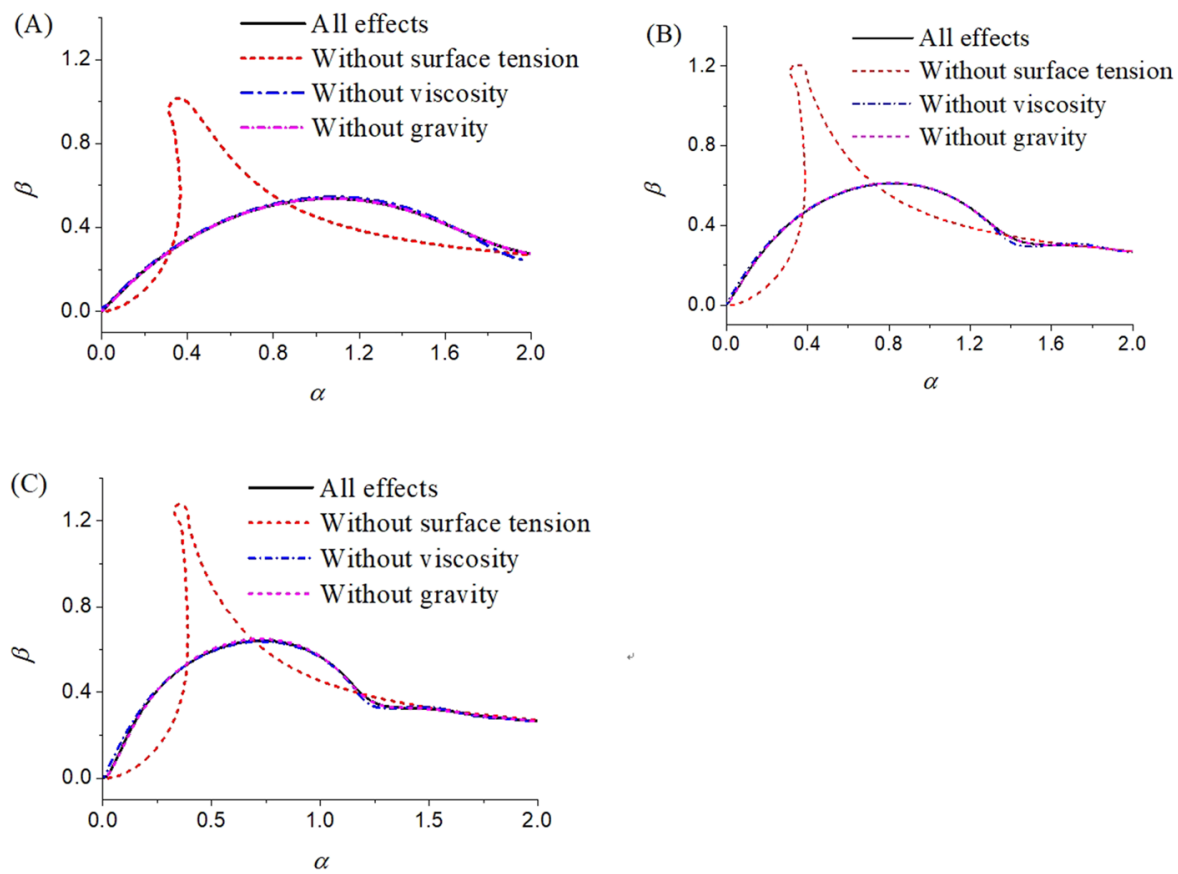

FIG. 8. The results for $(a=0.84, b=0.26)$ at different entry distances: (a) $s=0.01$ and $h / L=0.00471$, (b) $s=0.05$ and $h / L=0.00521$, and (c) $s=0.1$ and $h / L=0.0149$. 
approximation still gives a free surface shape very similar to that measured from the experiment.

\section{Impact of the plate into the mixture of water and glycerin}

As discussed at the beginning of Sec. III C, $a=2 / 3$ or $b=0$ corresponds to the impact with a constant velocity and the $R_{e}$ term may be more important than the $W_{e}$ term, depending on their values. We consider the case of the plate impact into the mixture of water and glycerin (58\%). We could set the dynamic viscosity coefficient $\mu=10 \mathrm{mPa}$ s, the surface tension coefficient $\sigma=0.068 \mathrm{~N} / \mathrm{m}^{2}$, and the water density $\rho=1150 \mathrm{~kg} / \mathrm{m}^{3}$, which is one of the cases in the work of Mayer and Krechetnikov. ${ }^{10}$ The half width of the plate $L=25 \mathrm{~mm}$, and the initial vertical velocity $V_{0}=0.25 \mathrm{~m} / \mathrm{s}$. In such a case, we have $W_{e}=4.794, R_{e}=718.75$, and $F_{r}=0.505$. Figure 7 gives the results at $s=0.01,0.05$, and 0.1 , which corresponds to $h / L=0.00471,0.00527$, and 0.0149. The simulation starts from $s_{0}=1 \times 10^{-4}$, which is much smaller than 0.01 , or the first instant at which the result is taken. As $c=0$ in this case, the gravity effect is of order $s^{2}$, which is small when $s$ is small unless $F_{r}$ is very low. This figure shows that at $F_{r}=0.505$, the gravity effect is insignificant. The effect of the viscous term is of order $s^{-1 / 2}$ and is, therefore, expected to be significant at small $s$ unless $R_{e}$ is very large. This figure shows that at $R_{e}=718.75$, the effect of the viscosity is to bend the jet to the left. The effect of the surface tension is of the zeroth order of $s$. Its significance will mainly depend on the value of $W_{e}$. This figure shows that at $W_{e}=4.794$, the sharp tip of the jet will be blunted with a crown appearing at the jet. The jet will bend to the right. Figure 7 , therefore, shows that at $b=0, W_{e}=4.794$, and $R_{e}=718.75$, the surface tension and viscosity effects are equally important at small $s$. The gravity effect at $F_{r}=0.505$ on the other hand is small.

We then consider the case with $c=0.31$ which corresponds to $a=0.84$ and $b=0.26$. The same $R_{e}$ and $W_{e}$ numbers as those in Fig. 7 will be used here. Figure 8 gives the free surface at $s=0.01,0.05$, and 0.1 . It can be seen that the surface tension has much more significant effects and the free surface is completely different when it is concluded. The viscous effect on the other hand is negligible, which is in sharp contrast to that in Fig. 7. We note that the exponents of $s$ for the $R_{e}$ and $W_{e}$ terms in this case are -0.81 and -0.62 , respectively, which are closer than -0.5 and 0 in Fig. 7 . As the Reynolds number is about 150 times the Weber number, the latter will become more significant, when their exponents of $s$ become closer. In Fig. 7, the viscous effect is mainly in the jet region. However, in Fig. 8, when the surface tension is included, the jet has been flattened. In such a case, the $R_{e}$ term no longer has a major effect.

\section{CONCLUSIONS}

The local flow near the edge of a horizontal plate with a length $2 L$ impacting a flat free surface with speed $V=V_{0}\left(t V_{0} / L\right)^{b}$ is investigated through velocity potential flow theory. From the extensive results obtained, the following conclusions can be drawn:

(1) In the coordinate system $(\alpha, \beta)=\left(\tilde{x} /\left(t V_{0} / L\right)^{a}, \tilde{y} /\left(t V_{0} / L\right)^{a}\right) /$ $(A \times L)$, when $a=2(b+1) / 3$, the solution is self-similar at any $a$ when the Weber number, Reynolds number, and Froude number are infinite and the effect of the vertical distance $h$ which the plate has traveled on the free surface is ignored. In such a case, the shape of the free surface is very sensitive to $b$. At small or negative $b$, fluid will be ejected with a jet bending toward the right. At larger $b$, the fluid will move to the left to fill the space left by the plate.

(2) The solution with the surface tension, viscous effect, and gravity effect can be self-similar when $a=2 / 3, a=1 / 2$, and $a=2$, respectively.

(3) Through the comparison with the experiment data undertaken by Mayer and Krechetnikov, ${ }^{10}$ it is found that scaling $a=0.71$ and $b=0.065$ in our numerical procedure fits the measured data best when the surface tension effect is dominant. The shape of the free surface matches the experimental data best still at $a=0.71$ and $b=0.065$ even when the viscous effect becomes more important.

(4) The significance of the $W_{e}, R_{e}$, and $F_{r}$ effects will depend on their relative values, the value of $b$, and time $t$.

\section{ACKNOWLEDGMENTS}

This work was supported by Lloyd's Register Foundation to which the authors are most grateful. Lloyd's Register Foundation helps to protect life and property by supporting engineering-related education, public engagement, and the application of research.

This work was also supported by the National Natural Science Foundation of China (Grant Nos. 51879123 and 51809123).

\section{DATA AVAILABILITY}

The data that support the findings of this study are available within the article.

\section{REFERENCES}

${ }^{1}$ Y. L. Yakimov, "Effect of the atmosphere with the fall of bodies into water," Fluid Dynamics 8(5), 679-682 (1973)

${ }^{2}$ A. Iafrati and A. A. Korobkin, "Initial stage of flat plate impact onto liquid free surface," Phys. Fluids 16, 2214 (2004).

${ }^{3}$ A. Iafrati and A. A. Korobkin, "Hydrodynamic loads during early stage of flat plate impact onto water surface," Phys. Fluids 20, 082104 (2008).

${ }^{4}$ B. W. Zeff, B. Kleber, J. Fineberg, and D. P. Lathrop, "Singularity dynamics in curvature collapse and jet eruption on a fluid surface," Nature 403, 401-404 (2000).

${ }^{\mathbf{5}}$ J. B. Keller, P. A. Milewski, and J.-M. Vanden-Broeck, "Merging and wetting driven by surface tension," Eur. J. Mech., B: Fluids 19, 491-502 (2000).

${ }^{6}$ D. Leppinen and J. R. Lister, "Capillary pinch-off in inviscid fluids," Phys. Fluids 15, 568-578 (2003).

${ }^{7}$ D. Vella and J. Li, "The impulsive motion of a small cylinder at an interface," Phys. Fluids 22, 052104 (2010).

${ }^{8}$ I. R. Peters, D. van der Meer, and J. M. Gordillo, "Splash wave and crown breakup after disc impact on a liquid surface," J. Fluid Mech. 724, 553-580 (2013).

${ }^{9}$ R. Krechetnikov, "Origin of ejecta in the water impact problem," Phys. Fluids 26, 052105 (2014).

${ }^{10}$ H. C. Mayer and R. Krechetnikov, "Flat plate impact on water," J. Fluid Mech. 850, 1066-1116 (2018).

${ }^{11}$ I. Robertson, S. J. Sherwin, and J. M. R. Graham, "Comparison of wall boundary conditions for numerical viscous free surface flow simulation," J. Fluids Struct. 19, 525-542 (2004).

${ }^{12} \mathrm{G}$. X. Wu and S. L. Sun, "Similarity solution for oblique water entry of an expanding paraboloid,” J. Fluid Mech. 745, 398-408 (2014). 
${ }^{13}$ S. Y. Sun, S. L. Sun, H. L. Ren, and G. X. Wu, "Splash jet and slamming generated by a rotating flap," Phys. Fluids 27, 092107 (2015).

${ }^{14} \mathrm{~J}$. Wang, O. M. Faltinsen, and C. Lugni, "Unsteady hydrodynamic forces of solid objects vertically entering the water surface," Phys. Fluids 31, 027101 (2019).
${ }^{15}$ C. M. Bao, G. X. Wu, and G. D. Xu, "Simulation of water entry of a twodimension finite wedge with flow detachment," J. Fluids Struct. 65, 44-59 (2016).

${ }^{16}$ Y. A. Semenov, G. X. Wu, and A. A. Korobkin, "Impact of liquids with different densities," J. Fluid Mech. 766, 5-27 (2015). 